\title{
Panorama da doença pulmonar obstrutiva crônica
}

\author{
An overview of chronic obstructive pulmonary disease
}

Renato Azambuja ${ }^{*}$

Mateus Bettencourt

Cláudia Henrique da Costa

Rogério Rufino

\section{Resumo}

A doença pulmonar obstrutiva crônica está em ascensão no mundo contemporâneo. É uma doença evitável e raramente atribuída à deficiência genética. Vários fatores são responsáveis por este aumento de incidência, como o tabagismo ativo e passivo, a poluição ambiental e ocupacional e a demora de implementação de políticas públicas eficazes para a sua prevenção. A prevalência tem grande variação no mundo - de $0,2 \%$ a $37 \%$-, e isto se deve, em parte, às características culturais dos países que emitem relatórios epidemiológicos, dos métodos de diagnóstico estabelecidos no diagnóstico e pela classificação da DPOC que foi utilizada. A prevalência e incidência é maior em homens, especialmente nos idosos acima de 75 anos de idade. A mortalidade aumentou nos últimos 30-40 anos e variou de 3 a 111 mortes por 100.000 habitantes. No Brasil, em 2011, o Instituto Nacional do Câncer publicou que $15,1 \%$ da população (de 190.732.694 milhões de pessoas) é fumante e que aproximadamente $15 \%$ irá desenvolver a
DPOC. Em números absolutos, 14.320 milhões brasileiros têm DPOC relacionada ao tabagismo. A DPOC é a quarta causa de morte nos Estados Unidos, o que representa 5\% dos óbitos totais, com um acréscimo de $8 \%$ do número total de mortes, de 116.494 para 126.005 , no período de 2000 a 2005. E, se a análise for estendida de 1980 a 2000, o aumento do número total de mortes corresponde a $67 \%$. Este capítulo tem como objetivo discutir a importância da doença como epidemia, que se caracteriza pelo aparecimento tardio e deterioração da função pulmonar muito lenta, mas que pode ser controlável por medidas mais rígidas do controle do tabagismo no Brasil.

Descritores: Doença pulmonar obstrutiva crônica; Tabagismo; Epidemiologia.

\footnotetext{
Abstract

Chronic obstructive pulmonary disease is on the rise in the contemporary World. It is a preventable disease and rarely attributed to genetic deficiency. Several factors are responsible

*Endereço para correspondência: Serviço de Pneumologia e Tisiologia, HUPE, UERJ. Boulevard 28 de Setembro, $77,2^{\circ}$ andar Rio de Janeiro, RJ, Brasil. CEP: 20551-030. E-mail: pneumo.uerj@gmail.com
} 
for this increased incidence, such as active and passive smoking, environmental pollution and occupational and delay of implementation of effective public policies for its prevention. The prevalence in the World varies greatly from $0.2 \%$ to $37 \%$, this wide range is explained in part by cultural characteristics of countries that emitting international epidemiological reports, methods used to establish the diagnosis and classification of COPD. The prevalence and incidence has being higher in men, especially in the elderly above 75 years of age. Mortality has also increased in the last 30 to 40 years, 3-111 deaths per 100,000 populations. In Brazil, in 2011, the National Cancer Institute reported that $15.1 \%$ of the population of $190,732,694$ million people in Brazil is smokers and about $15 \%$ of this population could develop the COPD. Thus, in absolute numbers, 4.32 million people have COPD Brazilian, only related to smoking. COPD is the fourth leading cause of death in the United States, representing $5 \%$ of all deaths, with an increase of $8 \%$ of the total number of deaths from 116,494 to 126,005 in the period from 2000 to 2005 . If the analysis is extended from 1980 to 2000 , the percentage of mortality was increased in $67 \%$. This chapter aims to demonstrate the importance of the epidemic, which is characterized by late onset and deterioration of lung function very slow, but that may be controllable by harsher measures of tobacco control in Brazil.

Keywords: Pulmonary disease, chronic obstructive; Smoking; Epidemiology.

\section{Conceito}

A doença pulmonar obstrutiva crônica (DPOC) é uma enfermidade respiratória prevenível e tratável, que se caracteriza pela presença de obstrução ao fluxo aéreo persistente ou parcialmente reversível. A obstrução do fluxo aéreo é geralmente progressiva e está associada a uma resposta inflamatória anormal dos pulmões decorrente da inalação de partículas ou gases tóxicos e tem consequências sistêmicas significativas. ${ }^{1} \mathrm{O}$ processo inflamatório crônico pode produzir alterações dos brônquios (bronquite crônica), bronquíolos (bronquiolite obstrutiva) e parênquima pulmonar (enfisema pulmonar). A predominância destas alterações é variável em cada indivíduo, tendo relação com os sintomas apresentados. Além do conceito fisiopatológico, a DPOC pode ser definida também pelos seus critérios diagnósticos funcionais: relação da $\mathrm{VEF}_{1}$ e CVF inferior a $70 \%$ na espirometria pós-broncodilatador. Tais critérios têm sido alvo de críticas de muitos estudiosos, visto que indivíduos saudáveis ao envelhecerem apresentam queda do $\mathrm{VEF}_{1}$ mais acentuada que da CVF, fazendo com que, muitas vezes, apresentem diminuição dessa razão. A mudança do critério diagnóstico para o uso do limite inferior da normalidade pode modificar a epidemiologia da doença, sobretudo nos indivíduos idosos.

\section{Epidemiologia}

Podemos dizer que o mundo vive uma epidemia global de DPOC. No passado o conceito de epidemia estava relacionado às doenças infecciosas, quando apresentavam um aumento agudo e inesperado na incidência. Este termo restrito da palavra não provinha exclusivamente da academia, mas da literatura coloquial. Na década de 90 , começou a se utilizar a expressão 'epidemia global' para caracterizar doenças ou estados metabólicos crônicos como diabetes mellitus, obesidade, hipertensão arterial sistêmica e também a DPOC. A ampliação do conceito foi essencial para o desenvolvimento de estratégias de prevenção e conscientização capitaneadas pela Organização Mundial de Saúde (OMS) e seus congêneres geopolíticos. ${ }^{2,3}$

A OMS considera que: 1) 65 milhões de pessoas no mundo têm DPOC de moderada a grave intensidade; 2) mais de 3 milhões de pacientes com DPOC morreram no ano de 2005; 3) em 2002, a DPOC foi a quinta causa de mortalidade mundial e 4) em 2020, será a terceira causa de mortalidade. Além disso, a DPOC é uma das principais causas de morbidade em todo o mundo. No Brasil, a prevalência da DPOC é 
estimada em 7,3 milhões de indivíduos (vide dados epidemiológicos no Brasil e Rio de Janeiro na tabela 1$){ }^{2,4}$

Como a epidemia de tabagismo acometeu primeiramente indivíduos do gênero masculino, a mortalidade entre homens ainda é superior em relação ao gênero feminino. Posteriormente, houve aumento da incidência do tabagismo entre as mulheres, o que justifica a ascensão da curva de mortalidade por DPOC no gênero feminino. Nos EUA a mortalidade em mulheres já superou a dos homens, mas não no Brasil. ${ }^{4}$

A principal etiologia da DPOC é o tabagismo ativo, cujo banimento seria suficiente para reduzir drasticamente o número de casos da doença. Durante muitos anos, considerou-se que apenas $15 \%$ dos fumantes desenvolveriam DPOC. Recentemente, demonstrou-se que a continuidade do tabagismo determinaria obstrução ao fluxo aéreo em até 50\% dos indivíduos com mais de 70 anos. ${ }^{5,6}$

\section{Estados Unidos}

Nos EUA, a DPOC é a quarta causa de mortalidade, representando $5 \%$ do total de mortes. No período de 2000 a 2005 houve um aumento de $8 \%$ do número total de mortes por DPOC - de 116.494 para 126.005. Se a análise for do ano de 1980 para 2000, o aumento do número total de mortes foi de $67 \%$. Além da expressiva elevação da taxa de mortalidade nos anos, o valor em dólares por paciente também foi um dos mais elevados (U\$ 6.300,00 e o tempo de permanência hospitalar médio foi de 8,18 dias, menor apenas que o de pacientes com doença renal crônica (9,51 dias). ${ }^{7,89}$

O controle de enfermidades prevalentes não infecciosas tem sido realizado com sucesso (figura 1). Apesar disso, a DPOC ainda encontra dificuldades de contenção do seu avanço. Parte desta dificuldade está no fato de que outros fatores de risco, além do tabagismo, estão sendo progressivamente relacionados à DPOC, como as infecções respiratórias em idades tenras, a história de asma, as novas exposições ocupacionais e o próprio envelhecimento do pulmão. ${ }^{1}$

A principal fonte de referência sobre a prevalência de DPOC nos EUA está disponível no estudo denominado NHANES III, que apresenta o resultado de um grande inquérito americano, com 7.429 pessoas, realizado entre os anos de 1988 a 1994. Os participantes tinham idades entre 25 e 75 anos e o estudo se baseou exclusivamente em valores obtidos pela espirometria. A DPOC de gravidade leve foi definida como $\mathrm{VEF}_{1} / \mathrm{CVF}$ menor que $70 \%$ e $\mathrm{VEF}_{1}$ maior ou igual a $80 \%$ do teórico previsto, representando $6,9 \%$ das pessoas. A DPOC moderada $\left(\mathrm{VEF}_{1} /\right.$ $\mathrm{CVF}<70 \%$ e $\mathrm{VEF}_{1} \leq 80 \%$ ) alcançou $6,6 \%$ dos participantes. A prevalência da DPOC, tanto leve quanto moderada, foi maior no sexo masculino do que no feminino, em brancos do que nos negros e aumentava acentuadamente com a idade. Independentemente da gravidade da do-

Tabela 1: Epidemiologia da DPOC no Brasil e no Rio de Janeiro.

\begin{tabular}{|c|c|c|c|c|c|c|c|}
\hline & Gênero & $\begin{array}{l}\text { População } \\
\text { (> 40anos) }\end{array}$ & Tabagistas & $\begin{array}{c}\text { DPOC } \\
\text { Classe I a IV }\end{array}$ & $\begin{array}{c}\text { DPOC } \\
\text { Classe II a IV }\end{array}$ & $\begin{array}{c}\text { Internação } \\
\text { no SUS } \\
\text { (2004) }\end{array}$ & $\begin{array}{c}\text { Óbitos por } \\
\text { DPOC } \\
\text { (2003) }\end{array}$ \\
\hline \multirow{3}{*}{ RJ } & Masculino & 2.122 .684 & 594.351 & 382.083 & & 6.319 & 2.079 \\
\hline & Feminino & 2.608 .213 & 521.642 & 365.149 & & 4.493 & 1.310 \\
\hline & Total & 4.730 .897 & 1.115 .993 & 747.232 & 269.661 & 10.812 & 3.390 \\
\hline \multirow{3}{*}{ Brasil } & Masculino & 21.867 .563 & 6.122 .917 & 3.936 .161 & & 105.883 & 20.713 \\
\hline & Feminino & 24.444 .017 & 4.888 .803 & 3.422 .162 & & 85.798 & 12.844 \\
\hline & Total & 46.311 .580 & 11.011 .720 & 7.358 .323 & 2.639 .760 & 191.681 & 33.560 \\
\hline
\end{tabular}

Fonte: Sociedade Brasileira de Pneumologia e Tisiologia. Disponível em: http: / /www.sbpt.org.br/downloads/arquivos/ Consenso_DPOC_SBPT_2006.pdf. Acessado em 9 de março de 2013. 
ença, menos de 50\% dos indivíduos com DPOC estavam cientes do seu diagnóstico. ${ }^{3}$

\section{América Latina}

Na América Latina, o estudo PLATINO (Projeto Latino Americano de Investigação em Obstrução Pulmonar), contou com a participação de diversos países da América Latina, dentre eles o Brasil. As cidades escolhidas para a investigação foram São Paulo (Brasil), cidade do México (México), Montevidéu (Uruguai) e Caracas (Venezuela). O estudo determinou a prevalência da DPOC, que variou de 7,8\% no México a $19,8 \%$ no Uruguai. No Brasil, a prevalência foi de $15,8 \%$ na cidade de São Paulo (figura 2). ${ }^{10,11,12}$

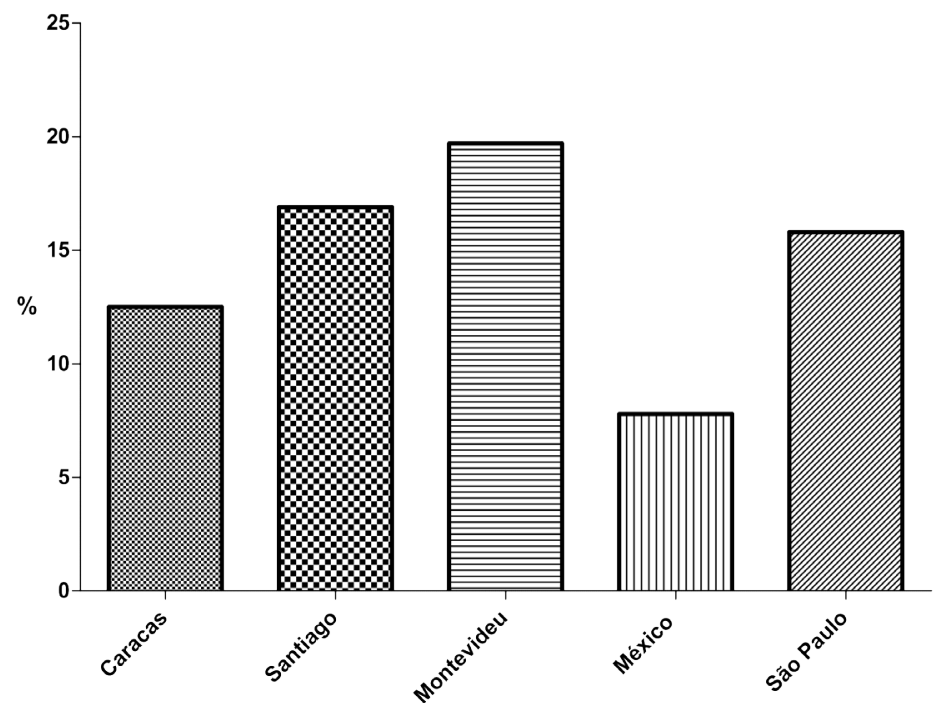

Figura 1: Evolução da DPOC nos Estados Unidos da América de 1965 até 1998. Fonte: http://www.who.int/respiratory/copd/burden/en/index.html. Acesso em 09 de março de 2013.

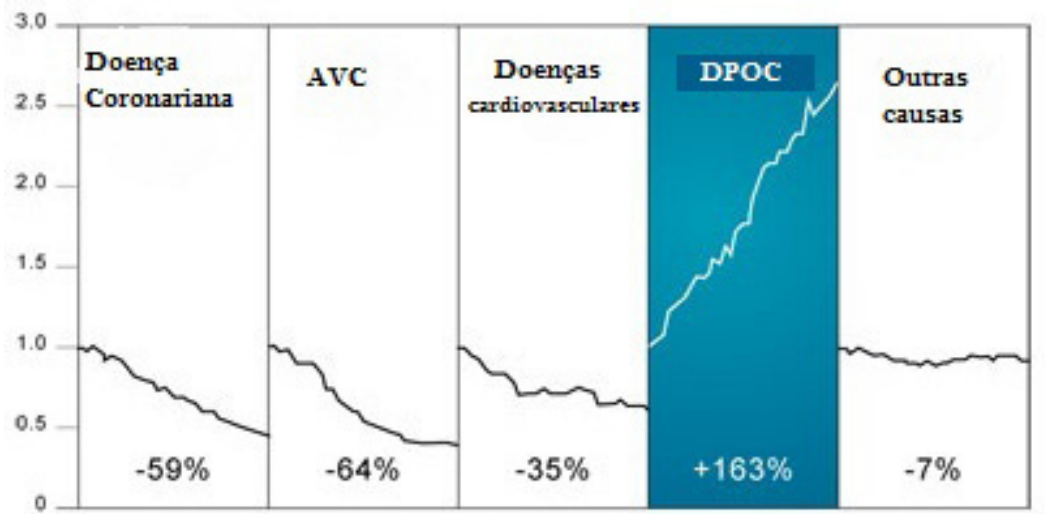

Figura 2: Frequência percentual de DPOC na população de grandes cidades da América Latina. Fonte: http://www.platino-alat.org. Acesso em 9 de março de 2013. 
No ano de 2011, o Instituto Nacional do Câncer publicou que $15,1 \%$ da população (de 190.732.694 milhões de pessoas) são tabagistas, o que representa o principal fator de risco para DPOC. Sabe-se que aproximadamente $15 \%$ destes desenvolvem a DPOC (figura 3). Logo, em números absolutos, haveria 4.320 .000 indivíduos com DPOC provenientes do tabagismo. ${ }^{6}$

\section{Custo da DPOC}

Em junho de 2004, Miravitles demonstrou o custo da DPOC nos países da América Latina. No Brasil, as despesas das visitas ambulatoriais, emergenciais e de internações foram, respectivamente, US\$ 25,00, US\$ 65,50 e US\$ 2.761,10. ${ }^{10}$ A Colômbia apresentava valores menores, tanto no atendimento ambulatorial quanto de urgência. Analisando a internação hospitalar, o custo anual da doença no Brasil por paciente foi um dos maiores de toda a América Latina, sendo superado somente pela Argentina (US\$ $3.120,00$ ), Equador (US\$2.800,00) e Venezuela (US\$ 3.086,00). Embora elevados, estes valores estão na realidade subestimados, pois não levam em consideração gastos indiretos como o absenteísmo no trabalho e a perda de vida produtiva, como aposentadoria precoce.

No trabalho de Sullivan e cols., do ano de 2000, a DPOC foi analisada comparativamente com outras doenças respiratórias de alta prevalência, utilizando dados divulgados pelo Instituto do Coração, Pulmão e Sangue dos Estados Unidos de 1993 (tabela 2). ${ }^{8}$ Por esta análise, verificou-se que a DPOC possui um

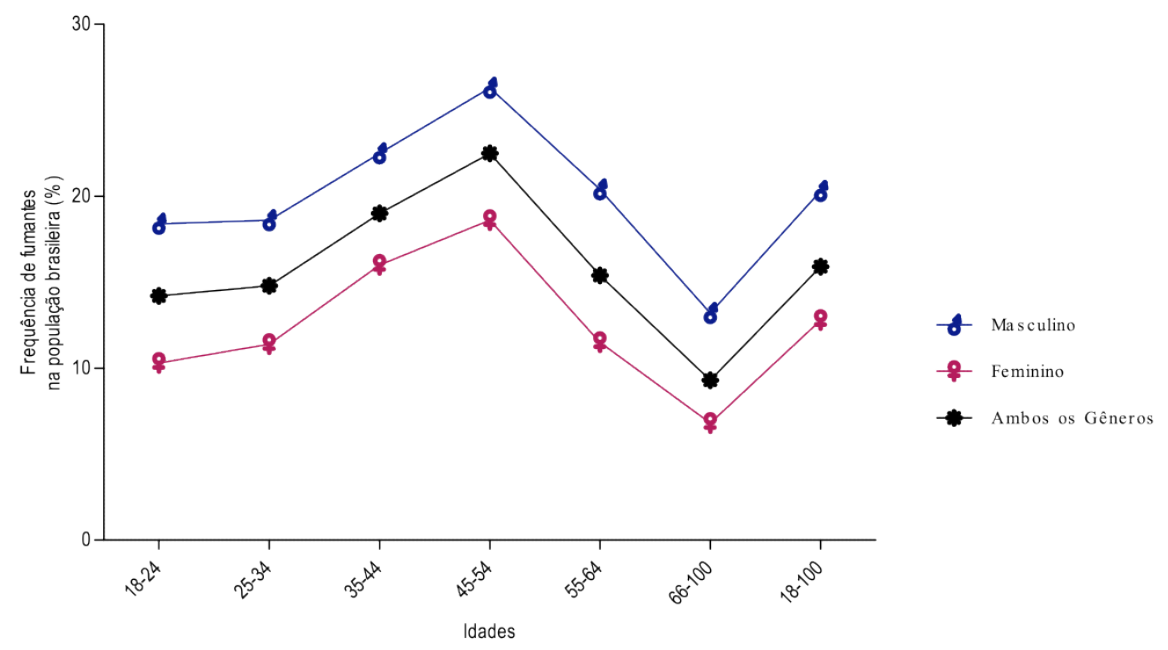

Figura 3: Frequência do tabagismo na população brasileira.

Fonte: https://apps.who.int/infobase/Indicators.aspx?ISO3=bra. Acessado em 9 de março de 2013.

Tabela 2: Análise comparativa do custo, mortalidade e morbidade das principais doenças respiratórias nos Estados Unidos.

\begin{tabular}{ccccc}
\hline Doenças/Aspectos & Custo total (\$) & $\begin{array}{c}\text { Custo médico } \\
\text { direto (\$) }\end{array}$ & Mortalidade (\%) & Morbidade (\%) \\
\hline DPOC & 23,9 & 14,7 & 4,5 & 4,7 \\
\hline Asma & 12,6 & 9,8 & 0,9 & 0,9 \\
\hline $\begin{array}{c}\text { Infecção pelo vírus } \\
\text { Influenza }\end{array}$ & 14,6 & 1,4 & 0,1 & 13,1 \\
\hline Pneumonia & 7,8 & 1,7 & 4,6 & - \\
\hline Tuberculose & 1,1 & 0,7 & - & 2,9 \\
\hline Câncer de pulmão & 25,0 & 5,1 & 17,1 & 1,5 \\
\hline
\end{tabular}


custo anual somente inferior ao do câncer de pulmão e que, apesar do alto esforço financeiro para o seu tratamento, a taxa de mortalidade é próxima das pneumonias, que utilizam valores $2 / 3$ menores.

\section{Conclusão}

A DPOC apresenta um elevado custo social e econômico à sociedade. Os pacientes são lentamente afastados do seu cotidiano em decorrência da diminuição de sua capacidade respiratória, até a temida necessidade de suplementação contínua de oxigênio domiciliar. As políticas governamentais de cessação do tabagismo são fundamentais para o possível controle da doença e devem ser estimuladas e ampliadas pela comunidade acadêmica.

\section{Referências}

1. GOLD. Guidelines Global Strategy for Diagnosis, Management, and Prevention of COPD [homepage on the Internet]. [updated 2013 December; cited 2012 november 1]. Available from:http://www.goldcopd.org/ uploads/users/files/GOLDReport_April112011. pdf.

2. Petty TL. The history of COPD. Int J Chron Obstruct Pulmon Dis. 2006;1(1):3-14.

3. World Health Organization. Chronic of respiratory disease. Burden of COPD. [homepage on the Internet]. [updated 2011; cited 2013 January 25]. Available from: http:// www.who.int/respiratory/copd/burden/en/ index.html.

4. CDC. Center for Disease Control and Prevention. National Health and Nutrition Examination Survey. [homepage on the Internet]. [cited 2012 November 30]. Available from: http://www.cdc. gov/nchs/nhanes/nh3data.htm.

5. Platino. Proyecto Latinoamericano de Investigación en Obstrucción. [homepage on the Internet]. [cited 2003 December 01]. Available from: http://www.platino-alat.org/.

6. INCA. Instituto Nacional do Câncer. [homepage on the Internet]. [cited 2012 Agosto 29]. Available from: http://www.inca.gov.br/ tabagismo/.

7. Sullivan SD, Ramsey SD, Lee TA. Economic burden of COPD. Chest. 2000; 117(2 Suppl):5S9S.

8. Naayan KM, Ali MK, del Rio C, et al. Global Noncommunicable Diseases-Lessons from the HIV-AIDS Experience. N Engl J Med. 2011;365(10):876.

9. Balkissoon R, Lommatzsch S, Carolan B, et al. Chronic obstructive pulmonary disease: a concise review. Med Clin North Am. 2011; 95 (6): 1125-41.

10. Miravitles M. Avaliação econômica de doença pulmonar obstrutiva crônica e de suas agudizações. Aplicação na América Latina. J Bras Pneumol. 2004;30(3):274-85.

11. CDC. Center for Disease Control and Prevention. Death from chronic obstructive lung disease, 2000-2005. [homepage on the Internet]. [updated 2008 cited 2008 November 14]. Available from: http://www.cdc.gov/ $\mathrm{mmwr} /$ preview/mmwrhtml/mm5745a4.htm.

12. Esteban-Vasallo MD, Dominguez-Berjon MF, Astray-Mochales J, Gènova-Maleras R, Pérez-Sania A, Sánchez-Perruca L, et al. Epidemiological usefulness of populationbased electronic clinical records in primary care: estimation of the prevalence of chronic diseases. Fam Pract. 2009;26(6):445-54. doi: 10.1093/fampra/cmp062.

13. Menezes AM, Victora CG, Rigatto M. Prevalence and risk factors for chronic bronchitis in Pelotas, RS, Brazil: a population-based study. Thorax. 1994;49(12):1217-21. 


\section{Autores}

\begin{abstract}
Abdiel Rolim
Programa de Residência Médica e Pós-graduação em Radiologia. Hospital Universitário Pedro Ernesto. Universidade do Estado do Rio de Janeiro. Rio de Janeiro, RJ, Brasil.
\end{abstract}

\section{Adalgisa I. M. Bromerschenckel}

Programa de Pós-graduação Stricto Sensu em Ciências Médicas. Faculdade de Ciências Médicas. Universidade do Estado do Rio de Janeiro. Rio de Janeiro, RJ, Brasil.

\section{Agnaldo José Lopes}

Disciplina de Pneumologia e Tisiologia. Departamento de Especialidades Médicas. Faculdade de Ciências Médicas. Universidade do Estado do Rio de Janeiro. Rio de Janeiro, RJ, Brasil.

\section{Ana Paula V. Soares}

Serviço de Pneumologia e Tisiologia. Policlínica Piquet Carneiro. Universidade do Estado do Rio de Janeiro. Rio de Janeiro, RJ, Brasil.

\section{Anamelia C. Faria}

Serviço de Pneumologia e Tisiologia. Hospital Universitário Pedro Ernesto. Universidade do Estado do Rio de Janeiro. Rio de Janeiro, RJ, Brasil.

\section{Domenico Capone}

Disciplina de Pneumologia e Tisiologia. Departamento de Especialidades Médicas. Faculdade de Ciências Médicas. Universidade do Estado do Rio de Janeiro. Rio de Janeiro, RJ, Brasil.

\section{Eduardo Costa F. Silva}

Serviço de Alergia e Imunologia. Departamento de Medicina Interna. Hospital Universitário Pedro Ernesto. Universidade do Estado do Rio de Janeiro. Rio de Janeiro, RJ, Brasil.

\section{Elizabeth J. C. Bessa}

Disciplina de Pneumologia e Tisiologia. Faculdade de Ciências Médicas. Universidade do Estado do Rio de Janeiro. Rio de Janeiro, RJ, Brasil.

\section{Gabriela A. C. Dias}

Serviço de Alergia e Imunologia. Departamento de Medicina Interna. Hospital Universitário Pedro Ernesto. Universidade do Estado do Rio de Janeiro. Rio de Janeiro, RJ, Brasil.

\section{Jorge Eduardo Pio}

Disciplina de Pneumologia e Tisiologia. Faculdade de Ciências Médicas. Universidade do Estado do Rio de Janeiro. Rio de Janeiro, RJ, Brasil.

\section{Kênia M. da Silva}

Programa de Pós-graduação Stricto Sensu em Ciências Médicas. Faculdade de Ciências Médicas. Universidade do Estado do Rio de Janeiro. Rio de Janeiro, RJ, Brasil.

\section{Leonardo P. Bruno}

Serviço de Pneumologia e Tisiologia. Hospital Universitário Pedro Ernesto. Universidade do Estado do Rio de Janeiro. Rio de Janeiro, RJ, Brasil.

\section{Lívia I. de O. Souza}

Faculdade de Ciências Médicas. Universidade do Estado do Rio de Janeiro. Rio de Janeiro, RJ, Brasil.

\section{Mateus Bettencourt}

Programa de Pós-graduação Stricto Sensu em Ciências Médicas. Faculdade de Ciências Médicas. Universidade do Estado do Rio de Janeiro. Rio de Janeiro, RJ, Brasil.

\section{Paulo Roberto Chauvet}

Disciplina de Pneumologia e Tisiologia. Faculdade de Ciências Médicas. Universidade do Estado do Rio de Janeiro. Rio de Janeiro, RJ, Brasil. 


\section{Rafael Capone}

Programa de Residência Médica e Pós-graduação em Radiologia. Hospital Universitário Pedro Ernesto. Universidade do Estado do Rio de Janeiro. Rio de Janeiro, RJ, Brasil.

\section{Renato Azambuja}

Serviço de Pneumologia e Tisiologia. Hospital Universitário Pedro Ernesto. Universidade do Estado do Rio de Janeiro. Rio de Janeiro, RJ, Brasil.

\section{Rogério M. Bártholo}

Programa de Pós-graduação Stricto Sensu em Ciências Médicas. Faculdade de Ciências Médicas. Universidade do Estado do Rio de Janeiro. Rio de Janeiro, RJ, Brasil.

\section{Sérgio da Cunha}

Disciplina de Tratamento Intensivo. Departamento de Clínica Médica. Faculdade de Ciências Médicas. Universidade do Estado do Rio de Janeiro. Rio de Janeiro, RJ, Brasil.

\section{Thiago P. Bártholo}

Serviço de Pneumologia e Tisiologia. Hospital Universitário Pedro Ernesto. Universidade do Estado do Rio de Janeiro. Rio de Janeiro, RJ, Brasil.

\section{Verônica S. Câmara}

Serviço de Pneumologia e Tisiologia. Policlínica Piquet Carneiro. Universidade do Estado do Rio de Janeiro. Rio de Janeiro, RJ, Brasil. 